\title{
POLYNOMIALS DEFINED BY GENERATING RELATIONS
}

\author{
BY \\ HARI BALLABH MITTAL
}

\begin{abstract}
Abstact. Various authors have made efforts for finding new generating functions for known polynomial sets. In the present paper, by making use of the operator $T_{k}=x(k+x D)$, a number of generating functions and characterizations have been obtained for various polynomials in a systematic manner.
\end{abstract}

1. Introduction. Various authors have made efforts for finding new generating functions for known polynomial sets. By means of purely manipulative skill some remarkably interesting results have been obtained.

Recently, in view of the results of Toscano [23], Brown [6] proved that for the Laguerre polynomials

$$
\sum_{n=0}^{\infty} L_{n}^{(a+m n)}(x) t^{n}=\frac{(1+v)^{a+1}}{1-m v} e^{-x v},
$$

where $v=t(1+v)^{m+1}, m$ being an integer. Also, assuming

$$
\sum_{n=0}^{\infty} L_{n}^{(a+m n)}(x) t^{n}=A(t) e^{x B(t)}
$$

he proved that

$$
\sum_{n=0}^{\infty} L_{n}^{(-a-(1+m) n)}(x) t^{n}=\frac{A(-t)}{1-B(-t)} \exp \left[\frac{-x B(-t)}{1-B(-t)}\right] .
$$

Carlitz [8] extended the results of Brown and showed that (1.1) and (1.3) hold for all values of $m$. In view of the results of Brown one may have under view the following question:

Can we obtain generating relations of the type (1.1) and (1.3) for other known polynomial sets and, in general, can we give a general method of obtaining such generating relations for special functions of interest?

The present paper is an answer to this question. By making use of the operational methods we obtain, in $\S 2$, an operational generating relation. We have proved, in

Received by the editors October 8, 1970 and, in revised form, August 2, 1971.

AMS 1970 subject classifications. Primary 33A65, 33A70; Secondary 05A15.

Key words and phrases. Generating functions, Laguerre polynomials, operational methods, Lagrange's expansion formula, operational generating formula, characterizations, formal power series, operational representations, generalized Laguerre polynomials, generalized Hermite polynomial, Bessel polynomial, Jacobi polynomial, Bedient's polynomial.

Copyright (C) 1972, American Mathematical Society 
$\S 3$, that generating relations of the type (1.1) and (1.3) are inherent to all special functions which are defined by (3.1). As illustrations, we obtain generating relations for the generalized Laguerre polynomials, generalized Hermite polynomials, Bessel polynomials and Jacobi polynomials. Further, in order to show the usefulness of our technique, a general class of polynomials has been considered, in $\S 9$, which incorporates the polynomials studied by Bedient [4], Sister Celine Fasenmyer [10], Rainville [20], Shively [22], Weisner [24] and others as special cases.

In this paper we have made use of the differential operator $T_{k}=x(k+x D), k$ being a constant (Mittal [14], [18]). It is easily seen that

$$
T_{k}^{n}\left\{x^{b+r}\right\}=(b+r+k)_{n} x^{b+r+n},
$$

where $n$ is a positive integer.

2. An operational generating relation. By making use of the Lagrange expansion formula

$$
(1+v)^{a+1}=1+(a+1) \sum_{n=1}^{\infty}\left(\begin{array}{c}
a+(b+1) n \\
n-1
\end{array}\right) \frac{t^{n}}{n}
$$

where $v=t(1+v)^{b+1}, v(0)=0$, Pólya and Szegö [19] showed that

$$
\frac{(1+v)^{a+1}}{1-b v}=\sum_{n=0}^{\infty}\left(\begin{array}{c}
a+(b+1) n \\
n
\end{array}\right) t^{n}
$$

where $v=t(1+v)^{b+1}, b$ being a constant.

Let $f(x)=\sum_{r=0}^{\infty} a_{r} x^{r}$ be uniformly convergent in $(\alpha, \beta)$ and let $b$ be a constant. Consider the sum

$$
\sum_{n=0}^{\infty} \frac{t^{n}}{n !} T_{(m-1) n+a+1}^{n}\left\{x^{b} f(x)\right\}=\sum_{n=0}^{\infty} \frac{t^{n}}{n !} T_{(m-1) n+a+1}^{n}\left\{\sum_{r=0}^{\infty} a_{r} x^{b+r}\right\}
$$

where $m$ is a constant.

Because term-by-term differentiation is justified, interchanging the order of summation, assumed to be justified, we have

$$
\begin{aligned}
\sum_{n=0}^{\infty} \frac{t^{n}}{n !} T_{(m-1) n+a+1}^{n}\left\{x^{b} f(x)\right\} & =\sum_{r=0}^{\infty} a_{r} x^{b+r} \sum_{n=0}^{\infty}(b+r+(m-1) n+a+1)_{n} \frac{x^{n} t^{n}}{n !} \\
& =\sum_{r=0}^{\infty} a_{r} x^{b+r} \sum_{n=0}^{\infty}\left(\begin{array}{c}
b+r+m n+a \\
n
\end{array}\right) x^{n} t^{n} .
\end{aligned}
$$

Making use of (2.2), in (2.3), we get

$$
\sum_{n=0}^{\infty} \frac{t^{n}}{n !} T_{(m-1) n+a+1}^{n}\left\{x^{b} f(x)\right\}=\sum_{r=0}^{\infty} a_{r} x^{b+r} \frac{(1+v)^{b+r+a+1}}{1-(m-1) v}
$$

where $v=x t(1+v)^{m}, m$ being a constant. Hence, we have the operational generating formula

$$
\sum_{n=0}^{\infty} \frac{t^{n}}{n !} T_{(m-1) n+a+1}^{n}\left\{x^{b} f(x)\right\}=\frac{x^{b}(1+v)^{a+b+1}}{1-(m-1) v} f[x(1+v)],
$$

where $v=x t(1+v)^{m}, m$ being a constant. 
It might be of interest to write the form of the result when $m$ is negative. From (2.1), by putting $b=m-1$, we have

$$
(1+v)^{a+1}=1+(a+1) \sum_{n=1}^{\infty}\left(\begin{array}{c}
a+m n \\
n-1
\end{array}\right) \frac{t^{n}}{n}
$$

where $v=t(1+v)^{m}, m$ a constant. Taking $a=0$ in (2.5), we get

$$
v=\sum_{n=1}^{\infty}\left(\begin{array}{c}
m n \\
n-1
\end{array}\right) \frac{t^{n}}{n}
$$

where $v=t(1+v)^{m}, m$ a constant. Similarly, for $a=-2,(2.5)$ gives

$$
\frac{v}{1+v}=\sum_{n=1}^{\infty}\left(\begin{array}{c}
m n-2 \\
n-1
\end{array}\right) \frac{t^{n}}{n}
$$

where $v=t(1+v)^{m}, m$ a constant. Define

$$
\begin{aligned}
B(t, c) & =-\sum_{n=1}^{\infty}\left(\begin{array}{c}
(c+1) n \\
n-1
\end{array}\right) \frac{t^{n}}{n} \\
A(t, a, c, d) & =(1-B(t, c))^{a+d+1} /(1+c B(t, c)) .
\end{aligned}
$$

In view of (2.6), (2.8) and (2.9), the relation (2.4) takes the form

$$
\sum_{n=0}^{\infty} \frac{t^{n}}{n !} T_{(m-1) n+a+1}^{n}\left\{x^{b} f(x)\right\}=x^{b} A(x t, a, m-1, b) f[x(1-B(x t, m-1))] .
$$

Again, making use of (2.7), it is easy to see that

$$
\begin{aligned}
B(t,-c-1) & =-B(-t, c) /(1-B(-t, c)), \\
A(t,-a,-c-1,-d) & =A(-t, a, c, d-1) \\
& =A(-t, a, c, d) /(1-B(-t, c)) .
\end{aligned}
$$

Therefore, by using (2.11) and (2.12) in (2.10), we have the operational formula

$$
\begin{aligned}
& \sum_{n=0}^{\infty} \frac{t^{n}}{n !} T_{-(m-1) n-a+1}^{n}\left\{x^{-b} f(x)\right\} \\
& =x^{-b} A(-x t, a, m-2, b-1) f[x /(1-B(-x t, m-2))],
\end{aligned}
$$

where $m$ is a constant. Again, if we use (2.11) and (2.13) in (2.10), we get the form

$$
\begin{aligned}
\sum_{n=0}^{\infty} \frac{t^{n}}{n !} T_{-(m-1) n-a+1}^{n}\left\{x^{-b} f(x)\right\} & \\
= & x^{-b} \frac{A(-x t, a, m-2, b)}{1-B(-x t, m-2)} f\left[\frac{x}{1-B(-x t, m-2)}\right] .
\end{aligned}
$$

The results in (2.14) and (2.15) are precisely the same.

3. Some characterizations. Let $f(x)$ admit a formal power series expansion $f(x)=\sum_{r=0}^{\infty} a_{r} x^{r}$, and, let

$$
T_{a+1}^{n}\{f(x)\}=n ! x^{n} g(x) f_{n}^{(a)}(x),
$$


where $T_{a+1}=x(a+1+x D), a$ being a constant and $g(x)$ being a function of $x$ alone. We have the following characterization for $f_{n}^{(a)}(x)$ :

THEOREM 1. A necessary and sufficient condition that $f_{n}^{(a)}(x)$ be defined by the generating relation

$$
\sum_{n=0}^{\infty} f_{a}^{(a+(m-1) n)}(x) t^{n}=[g(x)]^{-1} \frac{(1+v)^{a+1}}{1-(m-1) v} f[x(1+v)],
$$

where $v=t(1+v)^{m}$ and $m$ is a constant, is that it be given by (3.1).

Proof. The proof is simple and hence is omitted.

The generating function (3.2) can be rewritten in various other forms. As for example, let

$$
G(x, y(t))=[g(x)]^{-1} f[x y(t)],
$$

where $y(t)$ is some function independent of $x$. In view of (3.3), we can write (3.2) as

$$
\sum_{n=0}^{\infty} f_{n}^{(a+(m-1) n)}(x) t^{n}=\frac{(1+v)^{a+1}}{1-(m-1) v} G[x, 1+v],
$$

where $v=t(1+v)^{m}, m$ being a constant.

Again, if $F(x, t)$ is a function of $x$ and $t$, and if

$$
G(x, t)=F(x,(t-1) / t),
$$

then, from (3.4), we have

$$
\sum_{n=0}^{\infty} f_{n}^{(a+(m-1) n)}(x) t^{n}=\frac{(1+v)^{a+1}}{1-(m-1) v} F\left(x, \frac{v}{1+v}\right)
$$

where $m$ is a constant and $v=t(1+v)^{m}$. In particular, putting $m=1$ in (3.6), we immediately get

$$
\sum_{n=0}^{\infty} f_{n}^{(a)}(x) t^{n}=(1-t)^{-a-1} F(x, t)
$$

Again, putting $m=-1$ in (3.6), we get

$$
\sum_{n=0}^{\infty} f_{n}^{(a-2 n)}(x) t^{n}=(1+4 t)^{-1 / 2}\left[\frac{1+(1+4 t)^{1 / 2}}{2}\right]^{a+1} F\left[x, \frac{4 t}{\left(1+(1+4 t)^{1 / 2}\right)^{2}}\right],
$$

where $(1+4 t)^{1 / 2} \rightarrow 1$ as $t \rightarrow 0$. In view of Theorem 1 , we have the result:

THEOREM 2. A necessary and sufficient condition that $f_{n}^{(a)}(x)$ have a generating function of the form (3.8) is that it be given by (3.7).

A result similar to Theorem 2 has been derived by Brown [5]. A number of interesting special cases of Theorem 1 occur in the author's doctoral thesis [18] (see also [17]). 
Replacing $a$ by $-a$ and putting $m=-b$ in (3.6), we get

$$
\sum_{n=0}^{\infty} f_{n}^{(-a-(1+b) n)}(x) t^{n}=\frac{(1+v)^{1-a}}{1+(1+b) v} F\left(x, \frac{v}{1+v}\right),
$$

and, in view of Theorem 1, we have the following result:

THEOREM 3. A necessary and sufficient condition that $f_{n}^{(a)}(x)$ have a generating relation of the form

$$
\sum_{n=0}^{\infty} f_{n}^{(-a-(1+b) n)}(x) t^{n}=\frac{(1+v)^{1-a}}{1+(1+b) v} F\left(x, \frac{v}{1+v}\right)
$$

where $v(1+v)^{b}=t$ is that it be given by (3.7).

Recently Brown [7] also derived a result similar to that in Theorem 3.

4. A generalization of a result of Bailey. It is interesting to note that from the operational formula (2.4) one can easily derive a result due to Bailey [3]. In fact, putting $f(x)=1$ in (2.4), we get

$$
\sum_{n=0}^{\infty} \frac{(a+b+1)_{m n}}{(a+b+1)_{(m-1) n}} \frac{t^{n}}{n !}=\frac{(1+v)^{a+b+1}}{1-(m-1) v}
$$

where $v=t(1+v)^{m}, m$ being a positive integer now. Substituting the value of $t$ in terms of $v$, we get the result due to Bailey [3].

The operational formula (2.4) suggests an extension of (4.1). For, let $f(x)$ be defined by $f(x)=\sum_{r=0}^{\infty} a_{r} x^{r}$. Using the definition of the operator, we have

$$
\sum_{n=0}^{\infty} \frac{t^{n}}{n !} T_{(m-1) n+a+1}^{n}\left\{x^{b} f(x)\right\}=\sum_{n=0}^{\infty} \sum_{r=0}^{\infty} \frac{t^{n}}{n !} a_{r} \frac{(a+b+r+1)_{m n}}{(a+b+r+1)_{(m-1) n}} x^{b+r+n},
$$

where $m$ is a positive integer. Hence, from (2.4), we have

$$
\left.\begin{array}{rl}
\sum_{r=0}^{\infty} a_{r} x_{m}^{r} F_{m-1} & {\left[\begin{array}{ll}
\frac{a+b+r+1}{m}, \frac{a+b+r+2}{m}, \ldots, \frac{a+b+r+m-1}{m} ; & \frac{m^{m} v}{(m-1)^{m-1}(1+v)^{m}}
\end{array}\right]} \\
& =\frac{a+b+r+1}{m-1}, \frac{a+b+r+2}{m-1}, \ldots, \frac{a+b+r+m-1}{m-1} ;
\end{array}\right]
$$

where $m$ is a positive integer. If, in (4.3), we take $f(x)=$ const. (i.e., $a_{0} \neq 0$ and $a_{r}=0$ for $r \neq 0$ ) then it reduces to the result of Bailey [3]. In fact giving different values to the coefficients $a_{r}$, we get different relations of the form (4.3).

5. Generalized Laguerre polynomials. Earlier [13] we considered a generalization of the Laguerre polynomial, defined by

$$
T_{r n}^{(a)}(x)=n !^{-1} x^{-a} \exp \left\{p_{r}(x)\right\} D^{n}\left[x^{a+n} \exp \left\{-p_{r}(x)\right\}\right],
$$

where $p_{r}(x)$ is a polynomial in $x$ of degree not exceeding $r, a$ being a constant. In another communication [14] we proved that, for $m$ a constant,

$$
T_{m}^{n}\left\{x^{a-m+1} \exp \left\{-p_{r}(x)\right\}\right\}=n ! x^{a-m+1+n} \exp \left\{-p_{r}(x)\right\} T_{r n}^{(a)}(x),
$$


which gives a class of operational representations for the set of polynomials $T_{r n}^{(a)}(x)$. In particular, putting $m=a+1$ in (5.2), we obtained the result

$$
T_{a+1}^{n}\left\{\exp \left\{-p_{r}(x)\right\}\right\}=n ! x^{n} \exp \left\{-p_{r}(x)\right\} T_{r n}^{(a)}(x) .
$$

The polynomial $T_{r n}^{(a)}(x)$, defined in (5.1), reduces to generalized Laguerre polynomial of Chatterjea [9] for $p_{r}(x)=p x^{r}$ and to the Laguerre polynomial for $p_{r}(x)=x$.

Putting $b=0$ and $f(x)=\exp \left\{-p_{r}(x)\right\}$ in (2.4) and using (5.3) we immediately get the following generating function for the generalized Laguerre polynomial

$$
\sum_{n=0}^{\infty} T_{r n}^{(a+(m-1) n)}(x) t^{n}=\frac{(1+v)^{a+1}}{1-(m-1) v} \exp \left\{p_{r}(x)\right\} \exp \left\{-p_{r}[x(1+v)]\right\}
$$

where $v=t(1+v)^{m}, m$ being a constant and $p_{r}(x)$ a polynomial in $x$ of degree not exceeding $r$.

Also, putting $b=0$ and $f(x)=\exp \left\{-p_{r}(x)\right\}$ in (2.15), we get the result

$$
\begin{aligned}
\sum_{n=0}^{\infty} T_{r n}^{(-a-(m-1) n)}(x) t^{n} & \\
= & \frac{A(-t, a, m-2,0)}{1-B(-t, m-2)} \exp \left\{p_{r}(x)\right\} \exp \left\{-p_{r}\left[\frac{x}{1-B(-t, m-2)}\right]\right\}
\end{aligned}
$$

where $p_{r}(x)$ is a polynomial in $x$ of degree not exceeding $r, m$ is a constant and $B(t, c)$ and $A(t, a, c, d)$ are defined by (2.8) and (2.9) respectively.

In particular, putting $p_{r}(x)=x$ in (5.4), we get the following generating relation for the Laguerre polynomial

$$
\sum_{n=0}^{\infty} L_{n}^{(a+(m-1) n)}(x) t^{n}=\frac{(1+v)^{a+1}}{1-(m-1) v} e^{-x v},
$$

where $v=t(1+v)^{m}, m$ being a constant. Also, putting $p_{r}(x)=x$ in (5.5), we get the result

$$
\sum_{n=0}^{\infty} L_{n}^{(-a-(m+1) n)}(x) t^{n}=\frac{A(-t)}{1-B(-t)} \exp \left\{\frac{-x B(-t)}{1-B(-t)}\right\}
$$

where $A(-t)=A(-t, a, m, 0)$ and $B(-t)=B(-t, m), m$ being a constant. For $m$ a positive integer, the results in (5.6) and (5.7) were initially proved by Brown [6]. Carlitz [8] showed that (5.6) and (5.7) hold for all $\mathrm{m}$. A number of special cases of (5.4) and (5.5) have been derived in [18].

6. Generalized Hermite polynomials. As generalizations of the Hermite polynomial, we [18] considered a set of polynomials $\left\{H_{n}\left(x, a, p_{r}(x)\right)\right\}$, defined by the $n$th derivative formula

$$
H_{n}\left(x, a, p_{r}(x)\right)=(-1)^{n} x^{-a} \exp \left\{p_{r}(x)\right\} D^{n}\left[x^{a} \exp \left\{-p_{r}(x)\right\}\right],
$$

$p_{r}(x)$ being a polynomial in $x$ of degree not exceeding $r$. The polynomial in (6.1) reduces to the polynomial considered by Gould and Hopper [11] for $p_{r}(x)=p x^{r}$ 
and to the Hermite polynomial for $p_{r}(x)=x^{2}$ and $a=0$. Earlier [15] we proved that

$$
H_{n}\left(x, a, p_{r}(x)\right)=(-1)^{n} x^{m-n-a-1} \exp \left\{p_{r}(x)\right\} T_{m}^{n}\left\{x^{a-n-m+1} \exp \left\{-p_{r}(x)\right\}\right\},
$$

where $m$ is a constant. The relation in (6.2) gives a class of operational formulae for the generalized Hermite polynomial. In particular, for $m=a+1-n$, we get

$$
H_{n}\left(x, a, p_{r}(x)\right)=(-1)^{n} x^{-2 n} \exp \left\{p_{r}(x)\right\} T_{a+1-n}^{n}\left\{\exp \left\{-p_{r}(x)\right\}\right\},
$$

$p_{r}(x)$ being a polynomial in $x$ of degree not exceeding $r$.

Putting $b=0$ and $f(x)=\exp \left\{-p_{r}(x)\right\}$ in (2.4) and using (6.3) we immediately get the generating relation

$$
\sum_{n=0}^{\infty} \frac{t^{n}}{n !} H_{n}\left(x, a+m n, p_{r}(x)\right)=\frac{(1+v)^{a+1}}{1-(m-1) v} \exp \left\{p_{r}(x)\right\} \exp \left\{-p_{r}[x(1+v)]\right\},
$$

where $v=-(t / x)(1+v)^{m}, m$ being a constant. Also, putting $b=0$,

$$
f(x)=\exp \left\{-p_{r}(x)\right\}
$$

in (2.15), and using (6.3), we get the result

$$
\begin{aligned}
\sum_{n=0}^{\infty} \frac{t^{n}}{n !} H_{n}(x, & \left.-a-(m-2) n, p_{r}(x)\right) \\
& =\frac{A(t / x, a, m-2,0)}{1-B(t / x, m-2)} \exp \left\{p_{r}(x)\right\} \exp \left\{-p_{r}\left[\frac{x}{1-B(t / x, m-2)}\right]\right\}
\end{aligned}
$$

where $m$ is a constant. Putting $p_{r}(x)=p x^{r}$ in (6.4) and (6.5) one immediately gets the corresponding results for the generalized Hermite polynomial considered by Gould and Hopper [11]. A number of special cases of (6.4) have been given in [16], [18].

7. Bessel polynomials. Krall and Frink [12] considered a set of polynomials $\left\{y_{n}(a, b, x)\right\}$, defined by

$$
y_{n}(a+2, b, x)=b^{-n} x^{-a} e^{b / x} D^{n}\left[x^{2 n+a} e^{-b / x}\right] .
$$

The polynomial $y_{n}(a+2, b, x)$ is known as the Bessel polynomial. Al-Salam [1], [2] obtained a number of interesting results concerning Bessel polynomials. Earlier [15], [18], in making a study of these polynomials, we obtained the following class of operational representations for these polynomials

$$
T_{m}^{n}\left\{x^{n-m+a+1} e^{-b / x}\right\}=x^{n+a+1-m} e^{-b / x} b^{n} y_{n}(a+2, b, x),
$$

where $m$ is a constant. As a particular case, for $m=a+1+n$, we obtained the result

$$
T_{a+1+n}^{n}\left\{e^{-b / x}\right\}=b^{n} e^{-b / x} y_{n}(a+2, b, x) .
$$

Putting $b=0$ and $f(x)=e^{-b / x}$ in (2.4), and using (7.3), we immediately get the following generating relation for the Bessel polynomials

$$
\sum_{n=0}^{\infty} \frac{b^{n} t^{n}}{n !} y_{n}(a+(m-2) n+2, b, x)=\frac{(1+v)^{a+1}}{1-(m-1) v} \exp \left\{\frac{b v}{x(1+v)}\right\}
$$


where $v=x t(1+v)^{m}, m$ being a constant. Also, putting $b=0$ and $f(x)=\exp \{-b / x\}$ in (2.15), we get the interesting result

$$
\sum_{n=0}^{\infty} \frac{b^{n} t^{n}}{n !} y_{n}(-a-m n+2, b, x)=\frac{A(-x t, a, m-2,0)}{1-B(-x t, m-2)} \exp \left\{\frac{b}{x} B(-x t, m-2)\right\}
$$

where $m$ is a constant, $B(t, c)$ and $A(t, a, c, d)$ being defined by (2.8) and (2.9) respectively.

8. Jacobi polynomials. The Jacobi polynomial may be defined by the Rodrigues formula

$$
P_{n}^{(a, b)}(x)=\frac{(-1)^{n}(1-x)^{-a}(1+x)^{-b}}{n ! 2^{n}} D^{n}\left[(1-x)^{n+a}(1+x)^{n+b}\right] .
$$

Earlier [18], we obtained the following class of operational representations for Jacobi polynomials

$$
T_{m}^{n}\left\{x^{a+1-m}(1-x)^{n+b}\right\}=n !(1-x)^{b} x^{a+1+n-m} P_{n}^{(a, b)}(1-2 x),
$$

where $m$ is a constant. In particular, we obtained

$$
T_{a+1}^{n}\left\{(1-x)^{n+b}\right\}=n !(1-x)^{b} x^{n} P_{n}^{(a, b)}(1-2 x) .
$$

Putting $b=0$ and $f(x)=(1-x)^{b}$ in (2.4), and using (8.3), we get the following generating function for the Jacobi polynomials

$$
\sum_{n=0}^{\infty} P_{n}^{(a+(m-1) n, b-n)}(x) t^{n}=\frac{(1+v)^{a+1}}{1-(m-1) v}\left(\frac{1+x}{2}\right)^{-b}\left[1-\frac{(1-x)(1+v)}{2}\right]^{b},
$$

where $2 v=t(1+x)(1+v)^{m}, m$ being a constant. Also, putting $b=0$ and $f(x)=(1-x)^{b}$ in (2.15), and using (8.3), we get the result

$$
\begin{aligned}
\sum_{n=0}^{\infty} P_{n}^{(-a-(m-1) n, b-n)}(x) t^{n} \\
=\left(\frac{1+x}{2}\right)^{-b} \frac{A(-(t / x)(1+x), a, m-2,0)}{1-B(-t(1+x) / 2, m-2)} \\
\cdot\left[1-\frac{1}{2}\left\{\frac{1-x}{1-B(-t(1+x) / 2, m-2)}\right\}\right]^{b},
\end{aligned}
$$

where $m$ is a constant. Various special cases of (8.4) have been derived in [18].

9. Polynomials defined by generating relations. Let

$$
\psi(u)=\sum_{n=0}^{\infty} \gamma_{n} u^{n}, \quad \gamma_{0} \neq 0
$$

be a formal power series in $u$. Consider the power series

$$
\psi\left(c x^{p} t^{q}\right)=\sum_{n=0}^{\infty} \gamma_{n} c^{n} x^{p n} t^{n q},
$$


where $p$ and $q$ are positive integers and $c$ is a constant. Now putting $f(x)=\psi\left(c x^{p} t^{q}\right)$ and $b=0$ in (2.4), we immediately get

$$
\sum_{n=0}^{\infty} \frac{t^{n}}{n !} T_{(m-1) n+a+1}^{n}\left\{\psi\left(c x^{p} t^{q}\right)\right\}=\frac{(1+v)^{a+1}}{1-(m-1) v} \psi\left[c t^{q} x^{p}(1+v)^{p}\right]
$$

where $v=x t(1+v)^{m}, m$ being a positive integer. By making use of the definition of the operator $T_{k}$, we have

$$
\begin{aligned}
& \sum_{n=0}^{\infty} \frac{t^{n}}{n !} T_{(m-1) n+a+1}^{n}\left\{\psi\left(c x^{p} t^{q}\right)\right\} \\
& =\sum_{n=0}^{\infty} \sum_{r=0}^{\infty} \frac{t^{n+q r}}{n !} \gamma_{r} c^{r} T_{(m-1) n+a+1}^{n}\left\{x^{p r}\right\} \\
& =\sum_{n=0}^{\infty} \sum_{r=0}^{\infty} \frac{t^{n+q r}}{n !} \gamma_{r} c^{r}((m-1) n+a+1+p r)_{n} x^{p r+n} \\
& =\sum_{n=0}^{\infty} \sum_{r=0}^{[n / q]} \frac{t^{n}}{(n-q r) !} \gamma_{r} c^{r}(p r+(m-1) n-(m-1) q r+a+1)_{n-q r} x^{p r+n-q r} \\
& =\sum_{n=0}^{\infty} \frac{(a+1)_{m n}}{(a+1)_{m n-n}} \frac{x^{n} t^{n}}{n !} \sum_{r=0}^{[n / q]} \frac{(-n)_{q r}(a+1+m n)_{(p-m q) r}(-1)^{a r} \gamma_{r} c^{r} x^{(p-q) r}}{(a+1+(m-1) n)_{(p-(m-1) q) r}} \\
& =\sum_{n=0}^{\infty} \frac{(a+1)_{m n}}{(a+1)_{m n-n}} \frac{x^{n} t^{n}}{n !} \sum_{r=0}^{[n / q]} \frac{(-n)_{q r}(-a-m n+n)_{(m q-q-p) r} \gamma_{r} c^{r} x^{(p-q) r}}{(-a-m n)_{(m q-p) r}} \text {, }
\end{aligned}
$$

where $p \geqq m q$ in (9.3) and $p \leqq m q$ in (9.4), $m, p$ and $q$ being positive integers. Also, let

$$
f_{n, p, q}^{(a, m)}(c ; x)=\sum_{r=0}^{[n / q]} \frac{(-n)_{q r}(a+m n)_{(p-m q) r}}{(a+(m-1) n)_{(p-m q+q) r}}(-1)^{q r} \gamma_{r} c^{r} x^{(p-q) r}
$$

where $m, n, p$ and $q$ are positive integers. Using (9.5) in (9.3) we immediately get the relation

$$
\sum_{n=0}^{\infty} \frac{(a+1)_{m n}}{(a+1)_{m n-n}} f_{n, p, q}^{(a+1, m)}(c ; x) \frac{x^{n} t^{n}}{n !}=\frac{(1+v)^{a+1}}{1-(m-1) v} \psi\left(c t^{q} x^{p}(1+v)^{p}\right)
$$

where $v=x t(1+v)^{m}, m$ being a positive integer and $\psi(u)$ being a formal power series in $u$. Hence, we conclude that

"Corresponding to every power series $\psi(u)$, we can define a set of polynomials $\left\{f_{n, p, q}^{(a, m)}(c ; x)\right\}$, given by

$$
\sum_{n=0}^{\infty} \frac{(a+1)_{m n}}{(a+1)_{m n-n}} f_{n, p, q}^{(a+1, m)}(c ; x) \frac{x^{n} t^{n}}{n !}=\frac{(1+v)^{a+1}}{1-(m-1) v} \psi\left(c t^{q} x^{p}(1+v)^{p}\right),
$$

where $a$ and $c$ are constants, $p$ and $q$ are positive integers and $m$ is any integer and $v=x t(1+v)^{m}$."

It might be of interest to cite certain special cases of interest of the polynomials $f_{n, p, q}^{(a, m)}(c ; x)$ introduced in (9.5). Let

$$
\psi(u)={ }_{2} F_{1}\left(a^{\prime}, b^{\prime} ; c^{\prime} ; u\right) .
$$


With $\psi(u)$ as defined in (9.7), it is easily seen that, for $p=m=1,(9.5)$ becomes

$$
f_{n, 1, q}^{(a, 1)}(z ; x)={ }_{q+2} F_{q+1}\left[\begin{array}{l}
a^{\prime}, b^{\prime}, \frac{-n}{q}, \frac{1-n}{q}, \ldots, \frac{q-n-1}{q} ; \\
c^{\prime}, a, \frac{1-a-n}{q-1}, \ldots, \frac{q-a-n-1}{q-1} ;
\end{array}-\frac{z q^{q} x^{1-q}}{(q-1)^{q-1}}\right]
$$

and, using (9.6), we have the result

$$
\begin{aligned}
& \sum_{n=0}^{\infty} \frac{t^{n}}{n !}(a)_{n} x_{q+2}^{n} F_{q+1}\left[\begin{array}{l}
a^{\prime}, b^{\prime}, \frac{-n}{q}, \frac{1-n}{q}, \ldots, \frac{q-n-1}{q} ; \\
c^{\prime}, a, \frac{1-a-n}{q-1}, \ldots, \frac{q-a-n-1}{q-1} ;
\end{array}-\frac{z q^{q} x^{1-q}}{(q-1)^{q-1}}\right] \\
& =(1-x t)^{-a}{ }_{2} F_{1}\left(a^{\prime}, b^{\prime} ; c^{\prime} ; \frac{z x t^{q}}{1-x t}\right)
\end{aligned}
$$

where $q$ is a positive integer, $|x t|<1,\left|x z t^{q}\right|<1,\left|x z t^{q} /(1-x t)\right|<1$. If, however, we put $q=1$ in (9.8), then we get

$$
\sum_{n=0}^{\infty} \frac{t^{n}}{n !}(a)_{n 3} F_{2}\left[\begin{array}{r}
-n, a^{\prime}, b^{\prime} ; \\
a, c^{\prime} ;
\end{array}\right]=(1-t)^{-a}{ }_{2} F_{1}\left(a^{\prime}, b^{\prime} ; c^{\prime} ; \frac{-z t}{1-t}\right)
$$

which reduces to a result of Weisner [24] for $a=a^{\prime}$ and to a result of Rainville [21, p. 59] for $a=a^{\prime}$ and $z=1$.

Further, if in (9.8), we replace $x$ by $2 x$ and $2 z$ by $-1 / x$, then, for $q=2$, we get the result

$$
\begin{aligned}
\sum_{n=0}^{\infty} \frac{2^{n} x^{n} t^{n}}{n !}(a)_{n 4} F_{3}\left[\begin{array}{l}
a^{\prime}, b^{\prime},-n / 2,(1-n) / 2 ; 1 / x^{2} \\
c^{\prime}, a, 1-a-n ;
\end{array}\right. \\
=(1-2 x t)^{-a}{ }_{2} F_{1}\left[\begin{array}{r}
a^{\prime}, b^{\prime} ; \\
c^{\prime} ;
\end{array}-t^{2} /(1-2 x t)\right]
\end{aligned}
$$

which is a generalization of the polynomial $R_{n}$ introduced by Bedient [4]. In fact, putting $a=a^{\prime}=\beta, b^{\prime}=\gamma-\beta$ and $c^{\prime}=\gamma$ the polynomial in (9.10) immediately reduces to the Bedient's polynomial $R_{n}(\beta, \gamma ; x)$ and (9.10) to the relevant generating function (Rainville [21, p. 297]).

Again, if in (9.6), we take

$$
\psi(u)={ }_{r} F_{s}\left(a_{1}, a_{2}, \ldots, a_{r} ; b_{1}, b_{2}, \ldots, b_{s} ; u\right)
$$

where $r \leqq s+1$ and put $m=2, p=1$ and $c=z$ in (9.6), we get after some simple manipulations

$$
\sum_{n=0}^{\infty} \frac{(a)_{2 n}}{(a)_{n}} \frac{x^{n} t^{n}}{n !}{ }_{r+2 m-1} F_{s+2 m-1}
$$

9.11) $\left[\begin{array}{l}a_{1}, a_{2}, \ldots, a_{r}, \frac{-n}{q}, \frac{1-n}{q}, \ldots, \frac{q-1-n}{q}, \frac{1-a-n}{q-1}, \ldots, \frac{q-1-a-n}{q-1} ; \\ b_{1}, b_{2}, \ldots, b_{s}, \frac{1-a-2 n}{2 q-1}, \ldots, \ldots, \ldots, \frac{2 q-1-a-2 n}{2 q-1} ;\end{array}\right]$

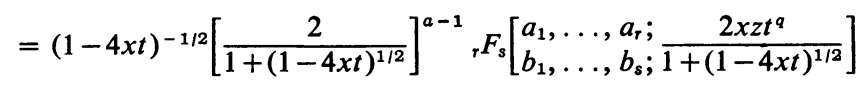


where $q$ is a positive integer and $r \leqq s+1$. The result in (9.11) is equivalent to a result of Rainville [20] (his (4)) for $q=1$. Again, putting $m=1, q=1$ in (9.6), it reduces to

$$
\sum_{n=0}^{\infty} \frac{t^{n}}{n !}(a)_{n} f_{n, p, 1}^{a, 1)}(c ; x)=(1-t)^{-a} \psi\left[\frac{c x^{p-1} t}{(1-t)^{p}}\right],
$$

where $a$ and $c$ are constants and $p$ a positive integer and $\psi(u)$ is defined by (9.1). Putting $p=2$ and $c=-4$ in (9.12), we get

$$
\sum_{n=0}^{\infty} \frac{t^{n}}{n !} f_{n, 2,1}^{(a, 1)}(-4 ; x)(a)_{n}=(1-t)^{-a} \psi\left[\frac{-4 x t}{(1-t)^{2}}\right] .
$$

The polynomial $f_{n, 2,1}^{(a, 1)}(-4 ; x)$, in $(9.13)$, has been considered (with a different notation) by Rainville [21, p. 137] and reduces to the class of polynomials $\left\{f_{n}(x)\right\}$ considered by Sister Celine Fasenmyer [10] for $a=1$. It may be remarked that by taking $m=1, q=1$ and

$$
\psi(u)={ }_{r} F_{s}\left(a_{1}, a_{2}, \ldots, a_{r} ; b_{1}, b_{2}, \ldots, b_{s} ; u\right),
$$

$r \leqq s+1$, in $(9.5)$, we get

$$
f_{n, p, 1}^{(a, 1)}(c ; x)={ }_{p+r} F_{q+s}\left[\begin{array}{l}
a_{1}, \ldots, a_{r}, \frac{a+n}{p-1}, \ldots, \frac{a+n+p-2}{p-1} ; \\
b_{1}, \ldots, b_{s}, \frac{a}{p}, \frac{a+1}{p}, \ldots, \frac{a+p-1}{p} ;
\end{array}-\frac{c[x(p-1)]^{p-1}}{p^{p}}\right]
$$

where $p$ is a positive integer. (9.14) reduces to Sister Celine's polynomial for $p=2$, $c=-4$ and $a=1$ (Rainville [21, p. 290]).

Lastly, putting $m=2, q=1$ and $c=z$ in (9.6), we immediately get

$$
\begin{aligned}
& \sum_{n=0}^{\infty} \frac{(a)_{2 n}}{(a)_{n}} f_{n, p, 1}^{(a, 2)}(z ; x) t^{n} \\
& \quad=(1-4 t)^{-1 / 2}\left[\frac{2}{1+(1-4 t)^{1 / 2}}\right]^{a-1} \psi\left[\frac{2^{p} x^{p-1} z t}{\left[\left(1+(1-4 t)^{1 / 2}\right]^{p}\right.}\right] .
\end{aligned}
$$

The polynomial $f_{n, p, 1}^{(a, 2)}(z ; x)$ in $(9.15)$ is a generalization of the pseudo-Laguerre polynomial considered by Shively [22]. In fact, taking $\psi(u)$ in (9.15) to be a generalized hypergeometric series and putting $p=2$ and $z=-1$ in (9.15), the polynomial reduces to the pseudo-Laguerre polynomial of Shively and the expression in (9.15) to the relevant generating relation (Rainville [21, p. 299]).

As is obvious, a number of other interesting special cases can be derived by giving particular values to the parameters in (9.6).

We shall, however, discuss some of the other applications of the operational formula (2.4) and derive other operational generating relations of the type (2.4) in subsequent communications.

My gratitude is due to Professor R. P. Agarwal for his kind guidance during the preparation of this paper. 


\section{REFERENCES}

1. W. A. Al-Salam, The Bessel polynomials, Duke Math. J. 24 (1957), 529-545. MR 19, 849.

2. - Some functions related to the Bessel polynomials, Duke Math. J. 26(1959), 519-539. MR $22 \# 120$.

3. W. N. Bailey, Transformations of generalized hypergeometric series, Proc. London Math. Soc. 29 (1929), 495-502.

4. P. E. Bedient, Polynomials related to Appell functions of two variables, Michigan Thesis, 1958.

5. J. W. Brown, On Burchnall's generating relation for Bessel polynomials, Amer. Math. Monthly 74 (1967), 1104-1107. MR 36 \#4034.

6. - On zero type sets of Laguerre polynomials, Duke Math. J. 35 (1968), 821-823. MR 38 \#2348.

7. - On the Sheffer A-type of certain modified polynomial sets, Proc. Amer. Math. Soc. 23 (1969), 718-722. MR 40 \#420.

8. L. Carlitz, Some generating functions for Laguerre polynomials, Duke Math. J. 35 (1968), 825-827. MR 39 \#1700.

9. S. K. Chatterjea, On a generalization of Laguerre polynomials, Rend. Sem. Mat. Univ. Padova 34 (1964), 180-190. MR 29 \#3697.

10. Sister M. Celine Fasenmyer, Some generalized hypergeometric polynomials, Bull. Amer. Math. Soc. 53 (1947), 806-812. MR 9, 184.

11. H. W. Gould and A. T. Hopper, Operational formulas connected with two generalizations of Hermite polynomials, Duke Math. J. 29 (1962), 51-62. MR 24 \#A2689.

12. H. L. Krall and O. Frink, A new class of orthogonal polynomials: The Bessel polynomials, Trans. Amer. Math. Soc. 65 (1949), 100-115. MR 10, 453.

13. H. B. Mittal, A generalization of Laguerre polynomials, Publ. Math. Debrecen (to appear).

14. - Operational representation for the generalized Laguerre polynomial, Glasnik Mat. Ser. III (to appear).

15. - Operational formulae for polynomials defined by a generalized Rodrigues formula, (to appear).

16. - Some generating functions, Rev. Fac. Ci. Univ. Lisboa 13 (1970), 43-54.

17. - On results involving generating functions and generalized hypergeometric function, Comment. Math. Univ. Carolinae (to appear).

18. - - A study of certain generating functions and associated polynomial sets, Lucknow University Thesis, Lucknow, India, 1970.

19. G. Pólya and G. Szegö, Aufgaben und Lehrsätze aus der Analysis. Vol. 1, 3rd ed., Die Grundlehren der math. Wissenschaften, Band 19, Springer-Verlag, Berlin, 1964. MR 30 \#1219a.

20. E. D. Rainville, Generating functions for Bessel and related polynomials, Canad. J. Math. 5 (1953), 104-106. MR 14, 872.

21. - Special functions, Macmillan, New York, 1960. MR 21 \#6447.

22. R. L. Shively, On pseudo Laguerre polynomials, Michigan Thesis, 1953.

23. L. Toscano, Funzioni generatrici di particolari polinomi di Laguerre e di altri da essi dipendenti, Boll. Un. Mat. Ital. (3) 7 (1952), 160-167. MR 14, 269.

24. L. Weisner, Group-theoretic origin of certain generating functions, Pacific J. Math. 5 (1955), 1033-1039. MR 19, 264.

Department of Mathematics, Lucknow University, Lucknow, India 